In addition, analysis for transcription factor motif enrichment in the differentially accessible peaks of the ATAC-Seq analysis revealed that motifs for TEAD2, RUNX family, and NFKB2 were less accessible, while motifs for JUN::FOS, NF2L2 and EHF were more accessible due to the chromatin changes. These results indicate that $\mathrm{H} 19 \mathrm{X}$ could promote or repress the activity of these candidate transcription factors by changing chromatin conformation at the sites of their consensus sequence.

Conclusion: ATAC-Seq in combination with microarray analysis identified candidate mechanism for the TGF $\beta$ regulated profibrotic effects of $\mathrm{H} 19 \mathrm{X}$ including direct effects on chromatin organization and on transcription factors associated with fibrotic pathways.

Disclosure of Interests: Elena Pachera: None declared, Shervin Assassi: None declared, Gloria Salazar: None declared, Mojca Frank-Bertoncelj : None declared, Jörg Distler: None declared, Gabriela Kania: None declared, Oliver Distler Grant/ research support from: Prof. Distler received research funding from Actelion, Bayer, Boehringer Ingelheim and Mitsubishi Tanabe to investigate potential treatments of scleroderma and its complications, Consultant for: Prof. Distler has/had consultancy relationship within the last 3 years with Actelion, AnaMar, Bayer, Boehringer Ingelheim, ChemomAb, espeRare foundation, Genentech/Roche, GSK, Inventiva, Italfarmaco, iQvia, Lilly, medac, Medlmmune, Mitsubishi Tanabe Pharma, Pharmacyclics, Novartis, Pfizer, Sanofi, Serodapharm and UCB in the area of potential treatments of scleroderma and its complications. In addition, he had/has consultancy relationship within the last 3 years with A. Menarini, Amgen, Abbvie, GSK, Mepha, MSD, Pfizer and UCB in the field of arthritides and related disorders

DOI: 10.1136/annrheumdis-2019-eular.5938

\section{OP0185 INFLAMMATION-DEPENDENT DECREASED EXPRESSION OF CD52 ON CIRCULATING CD14+ MONOCYTES FACILITATES ADHESION IN SYSTEMIC SCLEROSIS}

Michal Rudnik ${ }^{1}$, Mara Stellato ${ }^{1}$, Przemyslaw Blyszczuk ${ }^{1,2}$, Karin Klingel ${ }^{3}$, Jörg Henes ${ }^{4}$, Carol Feghali-Bostwick ${ }^{5}$, Oliver Distler ${ }^{1}$, Gabriela Kania ${ }^{1}$. ${ }^{1}$ Center of Experimental Rheumatology, University Hospital Zurich, Department of Rheumatology, Zurich, Switzerland; ' Jagiellonian University Medical College, Department of Clinical Immunology, Krakow, Poland; ${ }^{3}$ University Hospital Tuebingen, Institute for Pathology and Neuropathology, Tuebingen, Germany; ${ }^{4}$ University Hospital Tuebingen, Department of Rheumatology, Tuebingen, Germany; ${ }^{5}$ Medical University of South Carolina, Division of Rheumatology and Immunology, Charleston, SC, United States of America

Background: During the course of systemic sclerosis (SSc), infiltration of inflammatory cells, including monocytes, into the organs is a major process leading to fatal fibrosis, remodelling and organ dysfunction.

CD52 protein is highly expressed on CD4 ${ }^{+} \mathrm{T}$-cells and plays an important role in the modulation of T-cell receptor signalling. Nevertheless, the function of this protein on monocytes is not completely understood.

Objectives: We aimed to functionally investigate the role of circulating CD14 ${ }^{+}$ monocytes in the course of SSc with a special focus on monocyte adhesion and the influence of CD52 expression.

Methods: Biopsies from the heart, lungs and skin of SSc patients $(n=11,7,7$ respectively) and healthy controls $(H C)(n=10,7,9$ respectively) were analysed by immunohistochemistry for the presence of $\mathrm{CD} 14^{+}$cells. PolyA RNA sequencing of $\mathrm{CD}_{14}{ }^{+}$monocytes isolated from peripheral blood of IcSSc $(n=5$, age $=54.4$ $\pm 6.7)$, dcSSc patients $(n=5$, age $=51.8 \pm 7.2)$ and age- and sex-matched $\mathrm{HC}(n=5$, age $=50.8 \pm 9.7$ ) was performed using Illumina HiSeq 4000 platform. Differentially expressed genes were computed using DeSEQ2 algorithm. Gene ontology and pathway analysis were performed using Metacore software and ShinyApp. Expression of adhesion molecules was confirmed on the protein level using flow cytometry ( $\mathrm{HC} \mathrm{n}=8$, SSc $\mathrm{n}=22$ ). Adhesion of $\mathrm{CD}_{14}{ }^{+}$monocytes to immobilized ICAM1 and TNF $\alpha$-stimulated endothelial cells was checked using the 96-well plate adhesion assay $(\mathrm{HC} n=8, S S c n=22)$. CD52 regulation in $C D 14^{+}$monocytes from $\mathrm{HC}(n=4)$ was analysed on mRNA level upon stimulation with different concentrations of LPS, IFN $\gamma$, IL-4 and IL-13. THP-1 cell lines with overexpression and silencing of CD52 were generated using lentiviral vectors, and further, their adhesion was investigated $(n=4)$.

Results: Immunochemistry confirmed higher infiltration of $\mathrm{CD}_{14}{ }^{+}$cells in the heart $(p<0.01)$, lung $(p<0.05)$, and skin $(p<0.001)$ of SSc patients. We detected 1440 differentially expressed genes between dcSSc vs HC and 225 between IcSSc and HC CD14+ monocytes respectively ( $p \leq 0.01 ; \log 2$ ratio $\geq 0.5)$. Pathway analysis revealed significant alterations in processes important for tissue infiltration, including adhesion. Flow cytometry analysis showed upregulation of adhesion molecules: integrin $\alpha_{M}(C D 11 b)(p<0.01)$ and integrin $\beta_{2}(C D 18)(p<0.010 n$ the other hand, expression of CD52 was downregulated in SSc patients $(p<0.05)$. SSc CD14+ monocytes exhibited increased adhesion both to ICAM1-coated plates $(p<0.01)$ and to TNF $\alpha$-stimulated endothelial cells $(p<0.05)$. CD52 mRNA was significantly increased in a dose-dependent manner after anti-inflammatory stimulation (IL-4, IL-13) and significantly decreased after inflammatory stimulation (LPS, IFN- $\gamma)(p<0.05)$. Overexpression of CD52 in THP-1 monocytes decreased adhesion to TNF $\alpha$-stimulated endothelial cells $(p<0.01)$. Accordingly, silencing of CD52 increased adhesion of THP-1 monocytes $(p<0.01)$

Conclusion: This is the first report pointing to an increased adhesion of peripheral blood CD14 ${ }^{+}$monocytes to ICAM1 and endothelial cells in SSc. Our results suggest the primary activation of monocytes in peripheral blood, which translates into higher organ infiltration in SSc patients. Finally yet importantly, we characterised a novel function of CD52 molecule on monocytes and its possible contribution during the course of the disease.

Disclosure of Interests: Michal Rudnik: None declared, Mara Stellato: None declared, Przemyslaw Blyszczuk: None declared, Karin Klingel: None declared, Jörg Henes: None declared, Carol Feghali-Bostwick: None declared, Oliver Distler Grant/research support from: Prof. Distler received research funding from Actelion, Bayer, Boehringer Ingelheim and Mitsubishi Tanabe to investigate potential treatments of scleroderma and its complications, Consultant for: Prof Distler has/had consultancy relationship within the last 3 years with Actelion, AnaMar, Bayer, Boehringer Ingelheim, ChemomAb, espeRare foundation, Genentech/Roche, GSK, Inventiva, Italfarmaco, iQvia, Lilly, medac, Medlmmune, Mitsubishi Tanabe Pharma, Pharmacyclics, Novartis, Pfizer, Sanofi, Serodapharm and UCB in the area of potential treatments of scleroderma and its complications. In addition, he had/has consultancy relationship within the last 3 years with A. Menarini, Amgen, Abbvie, GSK, Mepha, MSD, Pfizer and UCB in the field of arthritides and related disorders, Gabriela Kania: None declared DOI: 10.1136/annrheumdis-2019-eular.5952

\section{OP0186 \\ LIN-GP38+ STROMAL CELLS ARE KEY EFFECTOR CELLS IN MYOCARDIAL FIBROSIS AND DEFECTS OF THE CONDUCTION SYSTEM}

Mara Stellato ${ }^{1}$, Michal Rudnik ${ }^{1}$, Florian Renoux ${ }^{1}$, Przemyslaw Blyszczuk $^{1,2}$ Elena Osto $^{3}$, Matthias Dewenter ${ }^{4}$, Petra Seebeck ${ }^{5}$, Elena Pachera ${ }^{1}$, Oliver Distler ${ }^{1}$, Gabriela Kania'. ${ }^{1}$ University Hospital Zurich, Department of Rheumatology, Zurich, Switzerland; ${ }^{2}$ Jagiellonian University Medical College,Krakow, Department of Clinical Immunology, krakow, Poland; ${ }^{3}$ University Hospital Zurich, Institute of Clinical Chemistry, Zurich, Switzerland; ${ }^{4}$ University Hospital Heidelberg, Department of Molecular Cardiology and Epigenetics, Heidelberg, Germany; ${ }^{5}$ University of Zurich, Zurich integrative Rodent Physiology, Zurich, Switzerland

Background: Cardiac dysfunction is a significant cause of mortality in SSc due to inflammation, vasculopathy and fibrosis. In fibrotic conditions, the excessive num ber of fibroblasts, myofibroblasts and extracellular matrix in the myocardium may directly or indirectly affect the cardiac conduction system by different mechanisms. Myofibroblasts are the main players in cardiac fibrogenesis, but their origin in SSc remains unknown.

Objectives: To unravel the role of Fos-related antigen 2 (Fra2) in stromal cell activation, cardiac fibrosis and alteration of the conduction system.

Methods: Fra2 transgenic (tg) mice and Rag2 ${ }^{-/}$Fra2 $\operatorname{tg}$ mice were studied Hearts from Fra2 tg and WT mice were analysed by immunohistochemistry (IHC) and immunofluorescence (IF). Cardiac function was assessed by echocardiography, electrocardiogram (ECG) and telemetry. Myocardial stroma

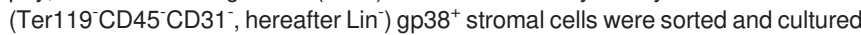
in vitro. The cellular phenotype was evaluated by qPCR, $\alpha$-smooth muscle actin $(\alpha \mathrm{SMA})$ and stress fibre staining, secreted collagens and contraction assay.

Results: Echocardiography of 20-week-old Fra2 tg mice displayed increased ejection fraction $(p=0.02)$ and fractional shortening $(p=0.02)$ with decreased left ventricle end-diameter and end-volume in systole $(p=0.006, p=0.008)$ and in diastole $(p=0.007, p=0.008)$. ECG in conscious mice revealed lower heart rate $(H R)$ (WT $741 \pm 33$ BPM, tg $644 \pm 37$ BPM, p<0.0001) and prolonged QRS, ST and QTc parameters $(p=0.001, p=0.01, p=0.01)$, which may predispose to arrhythmias. Telemetry recording showed the presence of atrio-ventricular (AV) blocks that correlated with disease severity $(R=0.9, p=0.02)$. The myocardium of Fra2 tg mice presented mild inflammation and fibrosis with higher expression of collagen $(p=0.03, n=7)$ and periostin $(p=0.001, n=7)$, increased number of $\alpha S^{+} A^{+}$myofibroblasts and expansion of stromal Lin-gp38 ${ }^{+}$cells compared to WT mice $(1.4 \% \pm$ $1.4, n=15$ and Fra- 2 tg: $6 \% \pm 4.5, n=14, p=0.001)$. Fibrosis disrupts the conductiv ity of cardiac tissue. Therefore, we investigated whether these stromal cells might be responsible for fibrosis. The majority of $\mathrm{Lin}^{-} \mathrm{gp} 38^{+}$cells co-expressed $\alpha \mathrm{SMA}$, collagen, ADAM12 and periostin, indicating that myocardial Lin ${ }^{-}{ }^{2} 38^{+}$cells might acquire a myofibroblast-like phenotype. Moreover, gp38 expression correlated with collagen deposition $(R=0.9, p<0.0001)$. The comparison between Lin ${ }^{-g} 38^{+}$ cells isolated from WT and Fra2 tg showed that both $\alpha$ SMA total protein and $\alpha$ SMA fibres were increased in Fra2 cells. Importantly, $\alpha$ SMA fibres co-localized with stress fibres, resulting in a faster and stronger contraction capacity of Fra2 cells $(p<0.0001, n=3)$. Proliferation of Fra2 cells was increased compared to WT $(p=0.007, n=5)$, while apoptosis was unchanged $(p=0.335, n=5)$. Interestingly, 